\title{
Phosphorylation et ciblage au protéasome : la F-box connection
}

D e l'étude des interactions protéiques entre le virus du SIDA et son hôte à celle de voies de signalisation fondamentales chez l'homme en passant par le développement chez la drosophile et le cycle cellulaire chez la levure Saccharomyces cerevisiae, il n'y a qu'un pas que l'étude de la $\beta \operatorname{TrCP}$, membre d'une nouvelle famille de protéines, les protéines à boîte $\mathrm{F}$ impliquées dans le ciblage des protéines au protéasome, nous a permis de franchir.

\section{Ubiquitinylation et dégradation par le protéasome}

Le protéasome 26S, particule cylindrique composée d'au moins 28 chaînes polypeptidiques différentes, est la principale machinerie de dégradation des protéines de la cellule en dehors des lysosomes. Il intervient dans la régulation de processus cellulaires fondamentaux, comme le cycle et la différenciation cellulaires, le développement, les voies de réparation de l'ADN, la régulation des réponses immunitaires et inflammatoires, l'apoptose, la modulation de l'expression de récepteurs de surface, la présentation des antigènes ou la transcription [1, 2]. Différentes sortes de protéasomes semblent participer à la dégradation des protéines impliquées dans ces activités. Les mécanismes de fonctionnement et de ciblage à ces différents protéasomes ne sont pas complètement élucidés. La grande variété des cibles moléculaires du protéasome nécessite une régulation et un contrôle temporel de la dégra-

enzymatiques complexes, d'événements de phosphorylation/déphosphorylation et d'association du substrat à différentes protéines, sont autant d'exemples de régulations possibles pour contrôler la vitesse de dégradation d'une protéine. L'ubiquitinylation, soumise aussi à de multiples niveaux de régulation, est en règle générale une étape qui précède la reconnaissance du substrat par le protéasome: il s'agit de l'attachement au substrat de plusieurs molécules d'ubiquitine, résultant de l'action concertée de trois activités enzymatiques appelées E1, E2 et E3 (figure 1) [3]. Schématiquement, E1 est l'enzyme d'activation qui lie l'ubiquitine de façon covalente en présence d'ATP, conduisant à une forme activée de l'ubiquitine. L'ubiquitine est ensuite transférée soit à E2, l'activité de conjugaison, puis à E3, l'activité ligase, puis au substrat, soit directement de E2 au substrat avec l'aide de protéines supplémentaires et adaptatrices. Alors que E1 est commune à toutes les voies de dégradation, n'ayant pour seule fonction que d'activer l'ubiquitine, les E3 ubiquitine ligases, qui interviennent dans la reconnaissance et donc dans le choix du substrat protéique à ubiquitinyler, et en conséquence à dégrader, jouent un rôle crucial dans la spécificité du processus de dégradation et sont donc extrêmement diverses en fonction de la nature de ces substrats. Cependant, en dépit de leur importance, peu de E3 ubiquitine ligases ont été décrites. Il s'agit de l'enzyme E3 $\alpha /$ UBR1 qui reconnaît en particulier des substrats répondant à la «règle de l'acide aminé amino-terminal " [2], du groupe des protéines contenant un domaine HECT (homologue de E6AP carboxy-terminal), dont le représentant E6-AP est nécessaire à la dégradation de p53 en présence de la protéine E6 du papillomavirus HPV (human papillomavirus) [3], du cyclosome encore appelé APC (anaphase promoting complex) impliqué notamment dans la dégradation de cyclines mitotiques [2, 4] et, enfin, des complexes SCF qui font l'objet de cet article.

I $\kappa B \alpha$ et la $\beta$-caténine sont dégradés par le protéasome

La régulation de deux voies de signalisation illustre l'importance du protéasome (figure 2): la voie d'activation de NFKB, mise en jeu au cours de multiples réactions immunitaires et inflammatoires $[5,6]$, et la voie $\mathrm{Wg} /$ Wnt chez la drosophile et chez l'homme, activée notamment au cours du développement, et de façon aberrante dans différents types de cancers [7-9]. L'activation du facteur de transcription NFKB est sous le contrôle des protéines inhibitrices de la famille IKB, et en particulier d'IאB $\alpha$ qui forme un complexe avec $\mathrm{NF \kappa B}$, le maintenant inactif dans le cytoplasme $\left(m / s ~ 1999, n^{\circ} 3, p .419 e t\right.$ 1999, $n^{\circ} 617, p$. 902). En réponse à un signal extracellulaire délivré par des cytokines comme le TNF $\alpha$ ou l'interleukine-1, IKB $\alpha$ est phosphorylée par un complexe kinase sur deux sérines spécifiques aux positions 32 et 36, puis ubiquitinylée et finalement dégradée par le protéasome [10]. NFKB migre alors dans le noyau 


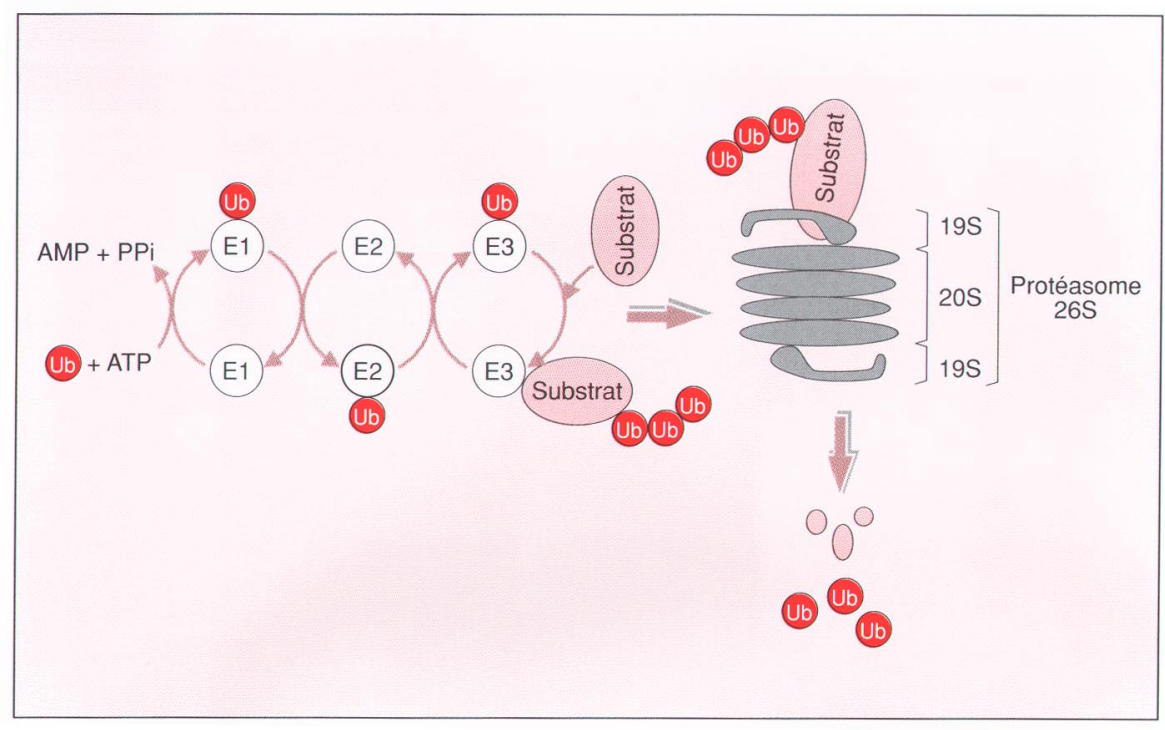

Figure 1. L'ubiquitinylation du substrat et la dégradation par le protéasome. La conjugaison de l'ubiquitine au substrat est réalisée successivement par les activités E1, E2 et E3; puis le substrat ubiquitinylé est dégradé par le protéasome 26S, composé du cœur catalytique $20 S$ et des deux particules régulatrices 19S. Une variante de ce schéma consiste à transférer l'ubiquitine directement de E2 au substrat avec l'aide de protéines supplémentaires, qui forment, avec I'enzyme E2, le complexe E3 ubiquitine ligase.

où il active la transcription de ses nombreux gènes cibles. A l'état constitutif, en l'absence de signal, $\mathrm{I} \kappa \mathrm{B} \alpha$ n'est pas phosphorylée, n'est pas reconnue par la machinerie d'ubiquitinylation et n'est donc pas dégradée.

Contrairement au facteur $\operatorname{I\kappa B} \alpha$, c'est à l'état constitutif, en l'absence de signal extracellulaire, que la $\beta$-caténine, protéine de la voie $\mathrm{Wg} / \mathrm{Wnt}$, est phosphorylée puis dégradée par le protéasome [11]. La dégradation de la $\beta$-caténine dépend de la phosphorylation de deux sérines en positions 33 et 37 de la protéine. Lorsque la voie est activée par un signal extracellulaire (facteur de la famille Wnt), la kinase responsable de la phosphorylation de la $\beta$-caténine est inactivée. La $\beta$-caténine, non phosphorylée, n'est plus dégradée. Elle s'accumule dans le cytoplasme puis migre dans le noyau où elle s'associe à des facteurs de la famille Tcf (T-cell factor)/LEF (lymphocyte enhancer factor) pour activer la transcription de gènes cibles, potentiellement oncogéniques comme $c$-myc. Cette voie de signalisation est essentielle pour le développement embryonnaire et le contrôle de la prolifération cellulaire $(\mathrm{m} / \mathrm{s} 1997$, téine APC (adenomatous polyposis coli), le suppresseur de tumeur fréquemment muté dans les cancers du côlon, et à l'axine [13]. Les mécanismes de phosphorylation d'IкB $\alpha$ et de la $\beta$-caténine étant élucidés, il restait à découvrir ceux responsables de l'ubiquitinylation des protéines phosphorylées. De façon surprenante, la découverte de l'activité E3 impliquée dans ces étapes d'ubiquitinylation a été permise, d'une part, par l'étude de la protéine $\mathrm{Vpu}$ du virus VIH-1 [14], et, d'autre part, par des études génétiques chez la drosophile [15].

\section{Comment la $\beta \operatorname{TrCP}$ humaine a été découverte}

Vpu est une petite protéine virale membranaire exprimée uniquement par le virus VIH-1, qui avec Nef et le précurseur de l'enveloppe du virus, la protéine gp160, contribue à la diminution de l'abondance du récepteur cellulaire $\mathrm{CD} 4$ à la surface des cellules infectées. Alors que Nef entraîne une accélération de l'endocytose de CD4 et sa dégradation ainsi que la rétention de CD4 au niveau du compartiment golgien tardif (TGN) [16], Vpu induit la dégradation de CD4 retenu au niveau du réticulum endoplasmique par son association avec la protéine gp160. La dégradation de CD4 permet la libération de la protéine d'enveloppe, qui subit une maturation protéolytique puis est incorporée dans les particules virales au niveau de la membrane plasmique. Outre sa fonction de dégradation de $\mathrm{CD} 4$, Vpu a aussi la capacité d'augmenter la production de particules virales par les cellules infectées [22].

Les deux fonctions biologiques connues de Vpu impliquent des domaines distincts de la protéine et sont liées à des états de phosphorylation différents. En effet, la phosphorylation de Vpu sur deux sérines (Ser 52 et Ser 56) est requise pour induire la dégradation de CD4 mais ne l'est pas pour l'augmentation de la production des virions. Alors que les travaux du laboratoire nous ont conduits à mettre en évidence une interaction entre Vpu et CD4, il est rapidement apparu que cette interaction ne suffisait pas pour déclencher la dégrada- 


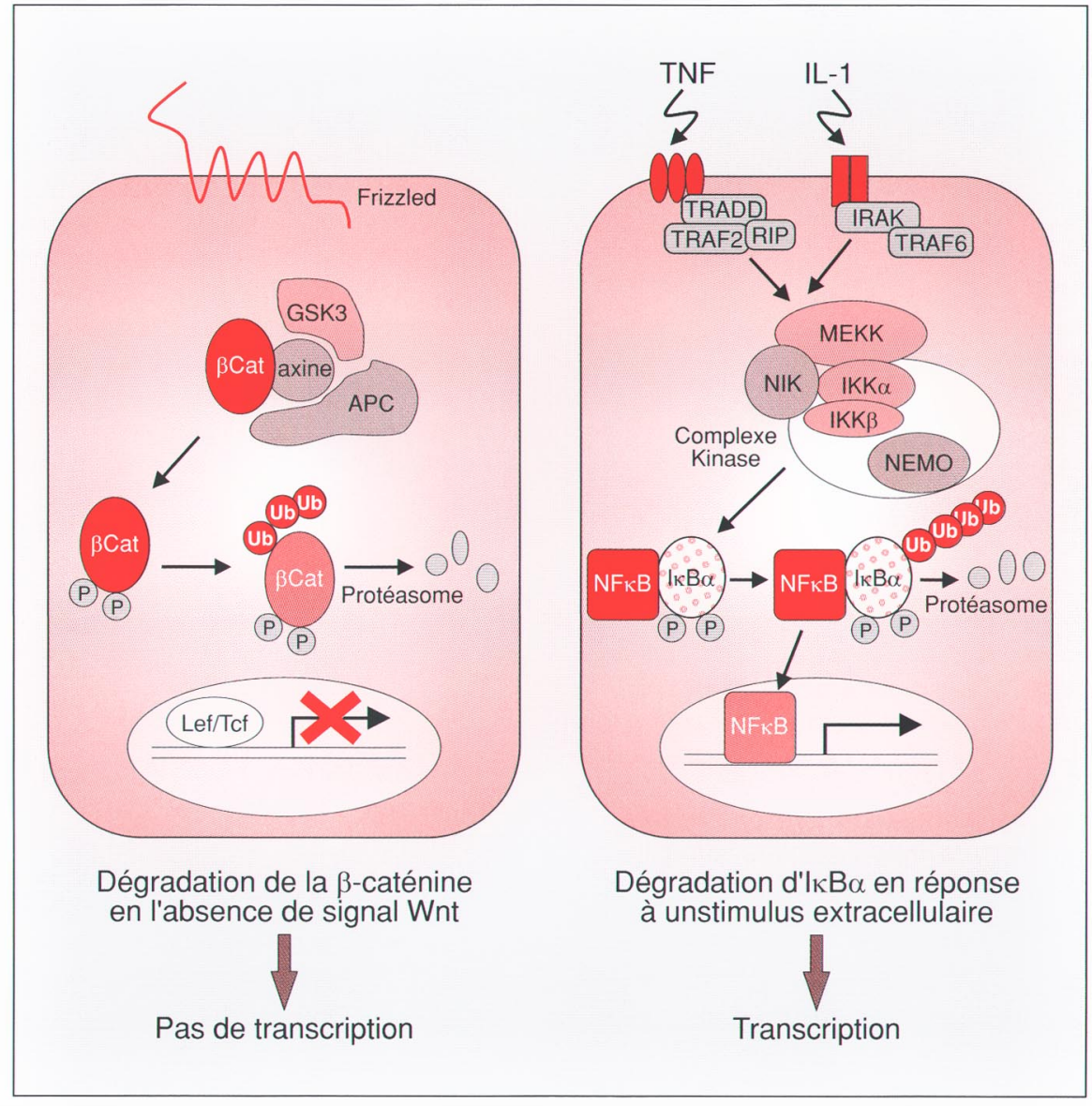

Figure 2. A. Inhibition de la voie de signalisation Wnt: dégradation de la $\beta$-caténine. De façon constitutive, la glycogène synthase kinase 3 (GSK3) phosphoryle la $\beta$-caténine en présence de l'axine et de APC. La $\beta$-caténine est ensuite ubiquitinylée et dégradée par le protéasome. La transcription des gènes cibles est alors inhibée. B. Activation de la voie de signalisation NFKB: dégradation $d^{\prime} \mid I B \alpha$. En réponse à un signal extracellulaire $(T N F \alpha, I L-1)$, le complexe de kinases permettant la phosphorylation de I $\mathrm{K} B \alpha$ est activé (complexe qui comprend MEKK, NIK, IKK- $\alpha$, IKK- $\beta$ et NEMO), IKB $\alpha$ est phosphorylée, ubiquitinylée puis dégradée. $N F \kappa B \alpha$ migre dans le noyau et active la transcription de gènes cibles.

tion de CD4. Notre but a été de comprendre comment Vpu utilise la machinerie cellulaire pour induire la dégradation de ce récepteur.

Le système du double-hybride, développé chez la levure Saccharomyces cerevisiae, est une méthode génétique performante pour mettre en évidence des interactions entre protéines. En utilisant cette approche sous la forme d'un crible génétique, nous avons identifié une nouvelle protéine cellulaire qui interagit avec Vpu [14]. Il s'agit d'une protéine homologue à une protéine de xénope, la $\beta \operatorname{TrCP}(\beta$ - d'une séquence appelée «boîte F», $\mathrm{du}$ fait de sa découverte, à l'origine, dans la cycline $\mathrm{F}$ [18] (figure 3A). Le motif «boîte $\mathrm{F}$ » a été identifié dans plus de 400 protéines après recherche dans des banques de données, mais seul un nombre très restreint de ces protéines a été caractérisé à ce jour (figure $3 B$ ). La boîte $\mathrm{F}$ interagit avec la protéine Skp1 et paraît principalement impliquée dans le ciblage de protéines au protéasome $[18,19]$.

Le rôle de la $\beta$ TrCP dans la dégradation de CD4 induite par Vpu est démontré par l'effet inhibiteur d'un mutant délété de la boîte $\mathrm{F}$ de la $\beta \operatorname{TrCP}(\beta \operatorname{TrCP} \Delta \mathrm{F})$ et contenant les motifs WD. Ce mutant a un effet "trans-dominant négatif» car il inhibe la dégradation de CD4 induite par Vpu. La dégradation de CD4 implique ainsi un réseau d'interactions : (1) Vpu interagit avec CD4 complexé à la protéine d'enveloppe au niveau de la membrane du réticulum endoplasmique; (2) Vpu recrute la $\beta$ TrCP au niveau de la membrane, grâce à une interaction avec les motifs WD. Cette interaction $\mathrm{Vpu} / \beta \mathrm{TrCP}$ dépend de la phosphorylation des sérines 52 et 56 de Vpu, expliquant au niveau moléculaire pourquoi cette phosphorylation est essentielle à la dégradation de CD4; (3) le domaine amino-terminal de la $\beta \operatorname{TrCP}$, contenant la boîte F, interagit avec Skp1; (4) Skp1 permet le recrutement de la $\beta$ TrCP dans un complexe qui assure le ciblage de CD4 vers le protéasome [14].

\section{De Vpu à la $\beta$-caténine et à $I \kappa B \alpha . .$.} en passant par la drosophile motifs répétés découverts à l'origine dans la transducine. Ces motifs, de 30 à 40 acides aminés, se terminent à l'extrémité carboxy-terminale par une séquence $\operatorname{Trp}(\mathrm{W})$-Asp(D) et sont ainsi appelés «motifs WD». Présents dans un grand nombre de protéines de fonctions variées, ils forment des surfaces qui favorisent les interactions entre protéines. Les motifs WD de la $\beta$ TrCP sont responsables de l'interaction avec la protéine $\mathrm{Vpu}$, qui est complexée à CD4 (figure 3A). A l'extrémité amino-terminale, la $\beta$ TrCP se caractérise par la présence
Le rôle de la $\beta$ TrCP en l'absence d'une infection par le virus VIH-1 restait à définir. Deux éléments nous ont récemment permis [20, 21], ainsi qu'à d'autres groupes [22-24], d'identifier des substrats cellulaires majeurs de la $\beta$ TrCP. Le premier élément est la séquence DSGXXS du motif de phosphorylation de Vpu. Ce motif, requis pour l'interaction avec la $\beta \operatorname{TrCP}$ et pour la dégradation de CD4, est remarquablement conservé dans la $\beta$-caténine et dans I $\kappa \mathrm{B} \alpha$ (figure 4A). De plus, il était établi que 


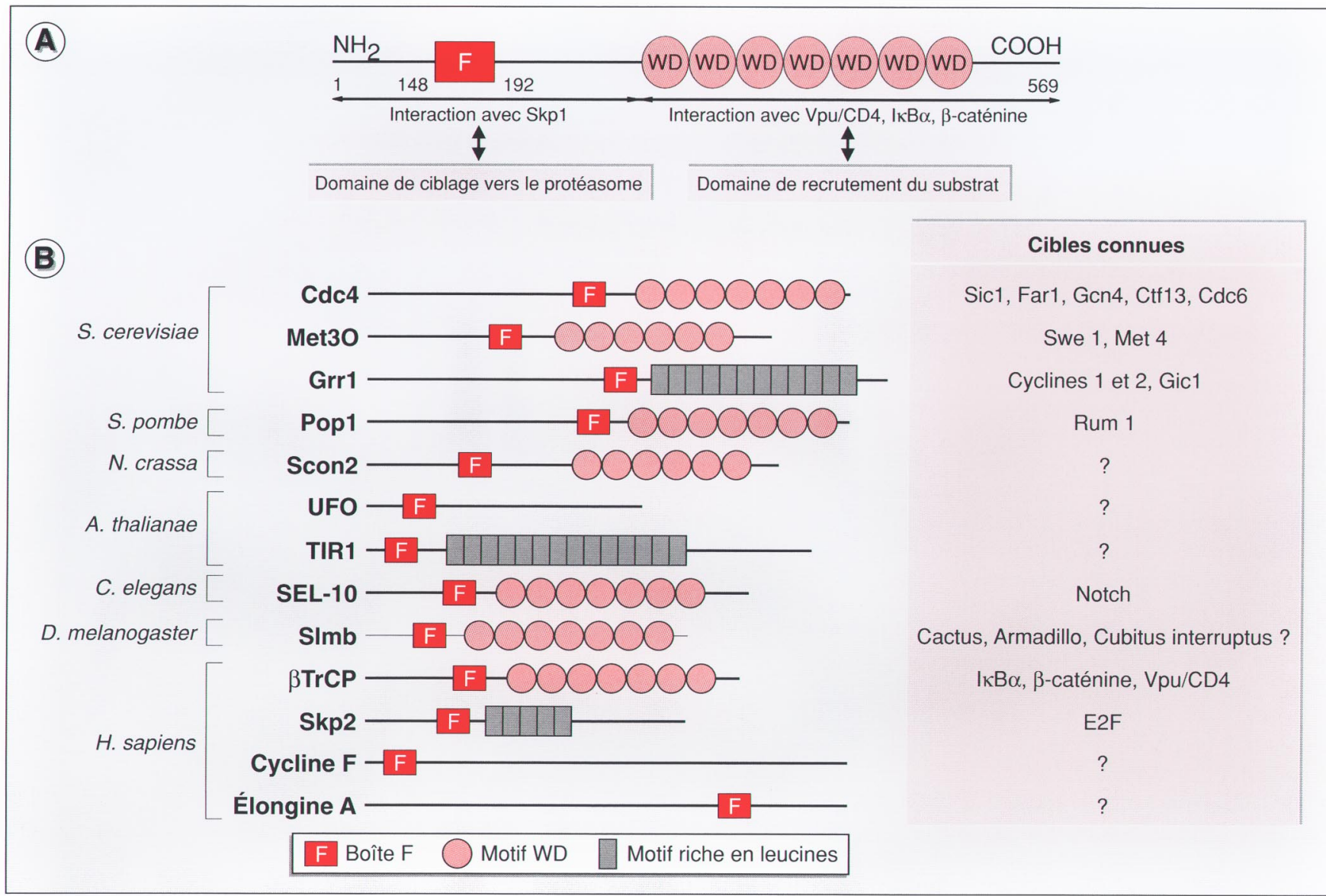

Figure 3. A. Structure et fonction de la $\beta$ TrCP. La $\beta$ TrCP possède une organisation bipartite: un domaine carboxy-terminal de recrutement du substrat qui interagit avec $V p u, I \kappa B \alpha$ et la $\beta$-caténine, et un domaine animo-terminal de ciblage vers le protéasome qui interagit avec Skp1. B. Les protéines à boîte $\boldsymbol{F}$. Les protéines à boîte $F$ sont présentes dans tout le règne animal (Met4, substrat de Met30 [18, 19]; E2F, substrat de Skp2 [29]). Elles participent à des fonctions variées: Cdc4, Grr1, Pop1, Skp2, la cycline F, Met 30 et Grr1 interviennent dans le contrôle du cycle cellulaire; Met30 est également impliqué dans le métabolisme de la méthionine, Grr1 dans l'expression des gènes stimulés par un signal glucose; Skp2 est impliqué dans la dégradation du facteur de transcription E2F, crucial pour le contrôle de la prolifération cellulaire; Scon2 et SEL-10 sont respectivement impliqués dans le métabolisme du soufre et la voie de signalisation Notch; UFO, TIR1 et Slimb interviennent au cours du développement; l'élongine A est une sousunité de l'élongine, impliquée dans l'élongation de la transcription.

la phosphorylation des sérines de ce motif était nécessaire à la dégradation de ces protéines par la voie ubiquitine/protéasome $[10,11]$.

Le deuxième élément est l'isolement de Slimb, l'homologue chez la drosophile de la $\beta \operatorname{TrCP}$ humaine, au cours d'un crible génétique visant à caractériser de nouveaux gènes de la morphogenèse de l'aile et de la patte [15]. L'étude moléculaire du mutant Slimb a révélé que la protéine Slimb est un régulateur négatif des voies Wingless (voie Wnt chez les vertébrés, figure 2) et Hedgehog, qui sont très conservées dans tout le règne animal. En effet, l'absence de Slimb entraîne l'accumulation d'Armadillo (l'homologue de la $\beta$-caténine humaine) et de la forme non tronquée de Cubitus Interruptus (facteur Ci, homologue des facteurs Gli humains), conduisant respectivement à la stimulation constitutive des gènes cibles des voies Wingless et Hedgehog.

Ainsi, ces deux éléments suggéraient que la $\beta \operatorname{TrCP}$ humaine pouvait permettre le ciblage de la $\beta$-caténine et de I $\mathrm{I} B \alpha$ vers le protéasome. Nous avons montré que le mutant de la $\beta \operatorname{TrCP}$ dépourvu de la boîte $\mathrm{F}$, $\beta \operatorname{TrCP} \Delta \mathrm{F}$, qui inhibe la dégradation de CD4 induite par Vpu, inhibe aussi la dégradation d'IкB $\alpha$ et de la $\beta$-caténine, entraînant l'inhibition de la transcription d'un gène rapporteur sous le contrôle de NFKB, et l'activation d'un autre gène rapporteur sous le contrôle d'un promoteur dépendant de la $\beta$-caténine $[20,24]$. Les interactions entre la $\beta \operatorname{TrCP}$ et $\mathrm{I} \kappa \mathrm{B} \alpha$ ou la $\beta$-caténine, détectées in vivo dans les cellules et dans le système double-hybride, dépendent, comme pour Vpu, de la phosphorylation des deux substrats sur les sérines du motif DSGXXS. C'est donc bien le 
même mécanisme qui est responsable du ciblage vers le protéasome de $\mathrm{Vpu} / \mathrm{CD} 4$, de IкB $\alpha$ et de la $\beta$-caténine. Le modèle $\mathrm{CD} 4 / \mathrm{Vpu} / \beta \operatorname{TrCP}$ se différencie par l'absence d'une liaison directe entre la $\beta \operatorname{TrCP}$ et le substrat qui est dégradé. Au cours d'une infection par le virus VIH-1, Vpu joue le rôle d'adaptateur entre la $\beta \operatorname{TrCP}$ et le récepteur $\mathrm{CD} 4$, pour induire la dégradation de ce dernier. Le mécanisme d'action de Vpu rappelle celui de la protéine E6 des papillomavirus (HPV). Dans les cellules infectées par un virus HPV, E6 joue le rôle d'adaptateur entre la protéine p53 et la protéine E6AP, une E3 ubiquitine ligase de type HECT. A la suite de la formation de ce complexe p53/E6/E6AP, la protéine p53 est ubiquitinylée puis dégradée par le protéasome [3].

Les complexes E3 ubiquitine ligase de type SCF : un mécanisme conservé de la levure à l'homme

La $\beta \operatorname{TrCP}$ a pu être caractérisée au sein de complexes E3 ubiquitine ligases de type SCF. L'activité d'ubiquitinylation de ces complexes a été mise en évidence sur des substrats tels que $\operatorname{I\kappa B} \alpha$ [22-24]. Les complexes «SCF » (S: Skp1; C: Cdc53 ou culline; F : protéine contenant une boîte F) ont été découverts chez la levure Saccharomyces cerevisiae, pour leur rôle dans la dégradation d'inhibiteurs du cycle cellulaire [19] (figure 4B). Le cour des complexes SCF comprend la protéine Skp1 et une protéine de la famille des cullines, Cdc53 chez la levure et la culline 1 chez l'homme, qui interagit directement avec Skp1. Ces deux protéines sont associées à un troisième partenaire, une protéine à boîte $\mathrm{F}$, telle la $\beta \operatorname{TrCP}$. Le prototype de la famille des protéines à boîte $\mathrm{F}$ est la protéine Cdc4 de Saccharomyces cerevisiae. Le domaine de recrutement du substrat dans les protéines à boîte F peut être composé de motifs WD (Cdc4 chez la levure, la $\beta$ TrCP chez l'homme) ou de répétitions leucine (Grr1 chez la levure, Skp2 chez l'homme), qui forment également des surfaces d'interactions protéiques (figure $3 B$ ). La protéine à boîte $\mathrm{F}$ confère sa spécificité et son nom au complexe SCF, parce qu'elle agit

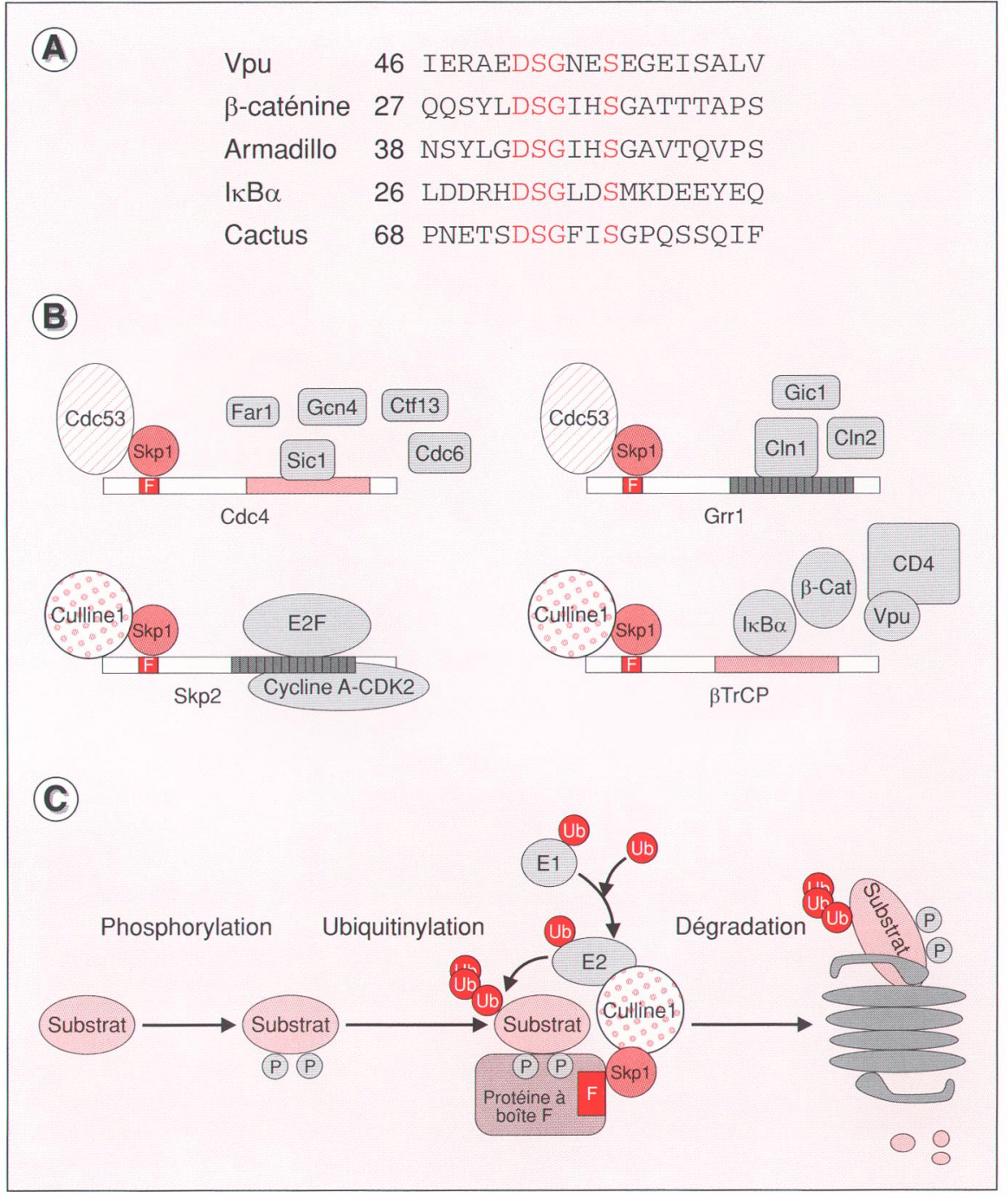

Figure 4. A. Le site consensus DSGXXS. Un motif identique essentiel pour I'interaction avec la $\beta \operatorname{TrCP}$ est présent dans la protéine Vpu du virus VIH-1, dans les protéines humaines $I_{\kappa} B \alpha$ et la $\beta$-caténine et leurs homologues chez la drosophile, Armadillo et Cactus. B. Les complexes SCF. Chez la levure Saccharomyces cerevisiae, les complexes $S_{C F}{ }^{C d c 4}$ et $S C F^{G r r 1}$ et, chez l'homme, les complexes SCF ${ }^{S k p 2}$ et $S^{\prime} C^{\beta T r C P}$ sont les mieux caractérisés. Chaque complexe recrute de manière spécifique plusieurs substrats (en rose). E2F est le seul substrat connu de Skp2; le complexe cyclineA-CDK2 est associé à Skp2, sans pour autant être dégradé [29]. C. Les étapes de la dégradation par le protéasome en présence d'un complexe SCF. La phosphorylation du substrat (sur les deux sérines du site consensus DSGXXS pour $I_{\kappa} B \alpha$ et la $\beta$-caténine) précède son ubiquitinylation. L'ubiquitine est ensuite transférée de E1 à E2 puis au substrat en présence du complexe SCF. Récemment, I'ubiquitinylation des protéines à boîte F, Cdc4 et Grr1, a également été mise en évidence. Enfin, le substrat ubiquitinylé est dégradé par le protéasome.

le recrutement des protéines à ubiquitinyler puis à dégrader par le protéasome (par exemple, SCF $^{\beta T r C P}$ ) (figure 4B).

Les complexes SCF sont associés à une enzyme E2 de conjugaison de l'ubiquitine. De nombreuses enzymes de type E2 ont été décrites, mais dans les complexes SCF caractérisés jusqu'à présent essentiellement chez la levure, l'enzyme E2 majoritairement impliquée est Cdc34. Des expériences de 
reconstitution in vitro ont permis de montrer que l'ubiquitinylation des protéines de levure Sicl et des cyclines 1 et 2, dont la dégradation contrôle la progression du cycle cellulaire, dépend de la phosphorylation de ces protéines, de la présence des enzymes E1, E2-Cdc34 et soit du complexe $\mathrm{SCF}^{\mathrm{Cdc} 4}$ dans lequel Cdc4 est la protéine qui recrute le substrat Sic1, soit du complexe $\mathrm{SCF}^{\mathrm{Grr}}$ qui recrute les cyclines [25, 26] (figures $4 B$ et $4 C$ ). Les protéines contenant des boîtes F, directement impliquées dans la reconnaissance des substrats, sont susceptibles d'adresser au protéasome des subtrats différents de manière très spécifique: ainsi la protéine Grr1 lie les cyclines 1 et 2 mais ne lie pas Sic1 tandis que la protéine Cdc4 interagit spécifiquement avec cet inhibiteur mais pas avec les deux cyclines.

Jusqu'à présent, les complexes SCF semblaient surtout impliqués dans le contrôle du cycle cellulaire, aussi bien chez la levure que chez l'homme. Le cas récent de la $\beta \operatorname{TrCP}$ montre que leur rôle est probablement beaucoup plus large. Comme nous l'avons discuté ci-dessus, la $\beta \operatorname{TrCP}$ est la protéine qui, au sein du complexe $\mathrm{SCF}^{\beta \operatorname{TrCP}}$, permet la reconnaissance spécifique des formes phosphorylées d'I $\mathrm{B} \alpha$ et de la $\beta$-caténine, conduisant à leur ubiquitinylation puis à leur dégradation par le protéasome (figure 4B). L'activité E2 $\mathrm{UBCH} 5$ peut s'associer in vitro au complexe SCF ${ }^{\beta \operatorname{TrCP}}$ [28]. Cependant, récemment, Gonen et al. [27] ont montré que plusieurs E2 (UBCH5b, UBCH5c et Cdc34) pouvaient permettre l'ubiquitinylation d'I $\mathrm{K} B \alpha$ [27]. La figure $4 C$ représente un schéma général des deux étapes - phosphorylation et ubiquitinylation qui caractérisent le ciblage d'une protéine au protéasome par un complexe de type SCF.

\section{$\beta$ TrCP, complexes de ciblage au protéasome et maladies}

Il est fort probable qu'un grand nombre de complexes de type SCF, responsables de nouvelles fonctions, seront prochainement découverts. Chez l'homme, il existe, en effet, non seulement une famille de protéines avec une boîte $\mathrm{F}$ mais aussi une famille de cullines. On peut donc supposer que, par une combinatoire de différentes E2 et de différents éléments des complexes SCF, une grande variété d'ubiquitine ligases peut être créée afin de répondre avec spécificité à l'ensemble des substrats à dégrader. La variété de ces enzymes est illustrée par la découverte des complexes VCB-like (VHL-Elongin C/Elongin B) $\left(m / s\right.$ 1999, $n^{\circ} 8-9$, p. 1051). Le prototype de cette nouvelle famille est le complexe entre la culline 2, l'élongine $\mathrm{B}$ et l'élongine $\mathrm{C}$ et le produit du gène $V H L$ (von Hippel Lindau), dont l'inactivation est responsable du syndrome VHL, une forme extrêmement rare de cancers héréditaires [28]. Le complexe élongine $\mathrm{BC} /$ culline $2 / \mathrm{VHL}$ a une structure semblable au complexe SCF, suggérant qu'il est également associé à une activité ubiquitine ligase [4]. Tout comme il existe une famille de complexes SCF, un ensemble de complexes VCB-like est actuellement mis en évidence. Dans ces complexes, VHL représente une famille de protéines qui contiennent une boîte SOCS (suppressor of cytokine signalling) et qui se lient à l'élongine BC. Ces protéines à boîte SOCS, comme les protéines à boîte F, seraient responsables du recrutement des substrats à dégrader. Les substrats de ces nouveaux complexes ne sont pas encore identifiés. Un facteur supplémentaire, commun aux complexes SCF et VCB-like, vient d'être découvert, la protéine Rbx1 [4]. Cette protéine jouerait un rôle dans le recrutement des E2 dans les E3 ubiquitine ligases. Il est intéressant de souligner que Rbx1 est l'homologue de la sous-unité Apc11, composant du complexe APG (anaphase promoting complex) [4]. Ainsi, l'architecture commune des complexes SCF, APC et VCB semble définir une «superfamille» d'E3 ubiquitine ligases [4].

La caractérisation de l'ensemble de ces complexes et des substrats qu'ils recrutent constituera une avancée majeure dans la compréhension des mécanismes régissant le ciblage d'une protéine au protéasome et pourrait aboutir à l'émergence de nouvelles cibles thérapeutiques. Cela est d'autant plus important qu'un lien a pu être établi entre de nombreuses maladies (cancers, maladies génétiques, maladies de Parkinson et d'Alzheimer, problèmes inflammatoires, infections virales...) et un mauvais fonctionnement de la cascade de l'ubiquitine et de la dégradation par le protéasome [2]. Comme nous l'avons mentionné, une dérégulation de l'expression de la voie Wnt et du taux de $\beta$-caténine est responsable de nombreux processus tumoraux [7-9] La $\beta \operatorname{TrCP}$ pourrait être un gène suppresseur de tumeurs dans la mesure où la $\beta \operatorname{TrCP}$ induit la dégradation de la $\beta$-caténine. De fait, un grand nombre de mutations oncogéniques de la $\beta$-caténine se retrouvent précisément dans le motif DSGXXS, crucial pour l'interaction avec la $\beta \operatorname{TrCP}$ $[8,9]$. La $\beta \operatorname{TrCP}$ apparaît donc comme une nouvelle cible thérapeutique très attractive pour la recherche de molécules anticancéreuses, de par son rôle vis-à-vis de la $\beta$-caténine, ou d'agents anti-inflammatoires, de par son rôle vis-à-vis de $\mathrm{I} \kappa \mathrm{B} \alpha$. Afin d'éviter des effets secondaires inappropriés, il sera nécessaire d'envisager le développement d'agents modulateurs extrêmement spécifiques de l'un ou de l'autre substrat

\section{RÉFÉRENCES}

1. Carillo S, Pariat M, Jariel-Encontre I, Steff AM, Piechaczyk M. Le catabolisme protéique intracellulaire: une fonction biologique majeure. Partie I. Med Sci 1995; 11 : 723-34.

2. Ciechanover A. The ubiquitin-proteasome pathway: on protein death and cell life. $E M B O J 1998 ; 17,24$ : 7151-60.

3. Scheffner M, Smith S, Jentsch S. The ubiquitin-conjugation system. In : Peters JM, Harris JR, Finley D, eds. Ubiquitin and the biology of the cell. New York: Raven Press, 1998.

4. Tyers M, Willems AR. One ring to rule a superfamily of E3 ubiquitin ligases. Science $1999 ; 284: 601-4$.

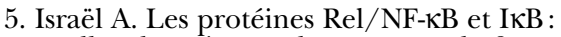
nouvelles données sur la structure, la fonction et la régulation. Med Sci 1995; 11 : 1017-20.

6. Baeuerle PA. Pro-inflammatory signalling: Last pieces in the NF-kB puzzle? Curr Biol 1998; 8: R19-22. 


\section{RÉFÉRENCES}

7. Cadigan KM, Nusse R. Wnt signalling: a common theme in animal development. Genes Dev 1997; 11 : 3286-305.

8. De la Coste A, Romagnolo B, Perret C. Une dérégulation de la voie de signalisation Wnt/ $\beta$-caténine impliquée dans l'hépatocarcinogenèse. Med Sci 1998; 14: 994-6.

9. Polakis $\mathrm{P}$. The oncogenic activation of $\beta$-catenin. Curr Opin Gen Dev 1999; 9 : 1521.

10. Stancovski I, Baltimore D. NF- $\kappa \mathrm{B}$ activation: the IкB kinase revealed? Cell 1997; 91: 299-302.

11. Willert K, Nusse R. $\beta$-catenin a key mediator of Wnt signalling. Curr Opin Gen Dev 1998 ; 8 : 95-102.

12. Yamaoka S, Courtois G, Bessia C, et al. Complementation cloning of NEMO, a component of the I $\kappa \mathrm{B}$ kinase complex essential for NF-кB activation. Cell 1998; 93 : 1231-40.

13. Hart MI, De Los Santos R, Albert IN, Rubinfeld B, Polakis P. Downregulation of $\beta$-catenin by human axin and its association with the APC tumor suppressor, $\beta$-catenin and GSK3 $\beta$. Curr Biol 1998; 8: R19-22.

14. Margottin F, Bour SP, Durand H, et al. A novel human WD protein, h- $\beta \operatorname{TrCP}$, that interacts with HIV-1 Vpu connects CD4 to the ER degradation pathway through an F-Box motif. Mol Cell 1998; 1: 565-74.

15. Jiang J, Struhl G. Regulation of the Hedgehog and Wingless signalling pathways by the F-box/WD40-repeat protein Slimb. Nature 1998; 391 : 493-6.

16. Piguet V, Gu F, Foti M, et al. Nef-induced CD4 degradation: a diacidic-based motif in Nef functions as a lysosomal targeting signal through the binding of $\beta$-COP in endosomes. Cell 1999; 97 : 63-73.

17. Lodge RE, Darlix JL, Cohen EA. L'infection par le VIH: rôle des facteurs viraux. Med Sci 1998; 14 : 148-54.

18. Bai C, Sen P, Hofmann K, et al. SKP1 connects cell cycle regulators to the ubiquitin proteolysis machinery through a novel motif, the F-Box. Cell 1996; 86: 263-74.

19. Patton EE, Willems AR, Tyers M. Combinatorial control in ubiquitin-dependent proteolysis : don't Skp the F-box hypothesis. Trends Genet 1998; 14, 6: 236-43.

20. Hart M, Concordet JP, Lassot I, et al. The F-box protein $\beta$ TrCP associates with $\beta$-catenin and regulates its activity in the cell. Curr Biol 1999; 9 : 207-10.

21. Kroll M, Margottin F, Kohl A, et al. Inducible degradation of I $\mathrm{KB} \alpha$ by the proteasome requires interaction with the F-box protein h- $\beta$ TrCP. I Biol Chem 1999; 274, 12 : 7941-5.

22. Yaron A, Hatzubai A, Davis M, et al. Identification of the receptor component of the IкB $\alpha$-ubiquitin ligase. Nature 1998; 396 : 590-4.

23. Spencer E, Jiang J, Chen ZJ. Signal-induced ubiquitinylation of $\mathrm{I} \kappa \mathrm{B} \alpha$ by the F-box protein Slimb/ $\beta$ TrCP. Genes Dev 1999; 13 : 284-94.

24. Winston JT, Strack P, Beer-Romero P, Chu CY, Elledge SJ, Harper JW. The SCF ${ }^{\beta T r C P}$ ubiquitin ligase complex associates specifically with phosphorylated destruction motifs in I $\kappa \mathrm{B} \alpha$ and $\beta$-catenin and stimulates $\mathrm{I} \kappa \mathrm{B} \alpha$ ubiquitinylation in vitro. Genes Dev $1999 ; 13$ : 270-83.

25. Renny Feldman RM, Correll CC, Kaplan KB, Deshaies RJ. A complex of Cdc $4 p$, Skplp, and Cdc53p/Culline catalyzes ubiquitinylation of the phosphorylated CDK inhibitor Siclp. Cell 1997; 91 : 221-30.

26. Skowyra D, Craig KL, Tyers M, Elledge SJ, Harper JW. F-Box proteins are receptors that recruit phosphorylated substrates to the SCF ubiquitin-ligase complex. Cell 1997; 91: 209-19.

27. Gonen H, Bercovich B, Orian A, et al. Identification of the ubiquitin carrier proteins, E2s, involved in signal-induced conjugation and subsequent degradation of I $\kappa \alpha$. J Biol Chem 1999 ; 274, 21 : 14823-30.

28. Pause A, Lee S, Worrell RA, et al. The von Hippel-Lindau tumor-suppressor gene product forms a stable complex with human CUL-2, a member of the Cdc53 family of proteins. Proc Natl Acad Sci USA 1997; 94: 2156-61.

29. Marti A, Wirbelauer C, Scheffner M, Krek W. Interaction between ubiquitin-protein ligase $\mathrm{SCF}^{\mathrm{SKP} 2}$ and E2F-1 underlies the regulation of E2F-1 degradation. Nat Cell Biol 1999; 1 : 14-9.

\section{Florence Margottin}

Chargée de recherche à l'Inserm

Irina Lassot

Étudiante en DEA de génétique moléculaire des maladies du développement et de l'oncogenèse

\section{Hervé Durand}

Assistant ingénieur à l'Inserm

\section{Richard Benarous}

Directeur du laboratoire «Interactions moléculaires hôte-pathogène ", EPI 9923 Inserm, Institut Cochin de génétique moléculaire, Université Paris V, 24, rue du Faubourg-Saint-Jacques, 75014 Paris, France.

\section{Mathias Kroll \\ Étudiant en thèse}

Françoise Bachelerie

Chargée de recherche à l'Inserm

Fernando Arenzana-Seisdedos

Directeur de recherche à l'Inserm

Unité d'immunologie virale, Institut Pasteur, 25 et 28, rue du Docteur-Roux, 75724 Paris Cedex 15, France.

\section{Jean-Paul Concordet}

Chargé de recherche à l'Inserm Inserm U. 129, Institut Cochin de génétique moléculaire, 24, rue du FaubourgSaint-Jacques, 75014 Paris, France.

\section{TIRÉS À PART}

F. Margottin. 